\title{
Film-Forming Properties of Fullerene Derivatives in Electrospray Deposition Method
}

\author{
Kazumasa Takeshi $^{1,2}$, Kenji Takagi ${ }^{1,2}$, Takeshi Fukuda ${ }^{1,2}$, Teiji Chihara ${ }^{1}$, Yusuke Tajima ${ }^{1,2^{*}}$ \\ ${ }^{1}$ Department of Functional Materials Science, Graduate School of Science and Engineering, Saitama University, Saitama, Japan; \\ ${ }^{2}$ RIKEN, Saitama, Japan. \\ Email: *tajima@riken.jp
}

Received November $27^{\text {th }}, 2012$; revised December $28^{\text {th }}, 2012$; accepted January $4^{\text {th }}, 2013$

\begin{abstract}
Thin films of three types of fullerene derivatives were prepared through the electrospray deposition (ESD) method. The optimized conditions for the fabrication of the thin films were investigated for different types of fullerene derivatives: $[6,6]$-phenyl- $\mathrm{C}_{61}$-butyric acid methyl ester, [6,6]-phenyl- $\mathrm{C}_{71}$-butyric acid methyl ester, and indene- $\mathrm{C}_{60}$-monoadduct. The spray diameter during the ESD process was observed as a function of the supply rate achieved by changing the applied voltage. In all cases, the spray diameter increased with increasing applied voltage, reaching the maximum diameter $\left(D_{\max }\right)$ in the voltage range 4 to $6 \mathrm{kV}$. It was clear that $D_{\max }$ was influenced by the dipole moments of the fullerene derivatives (as calculated by density functional theory methods). Scanning electron microscopy observation of the fabricated thin films showed that imbricated structures were formed through the stacking of the fullerene-derivative sheets. Atomic force microscopy images revealed that the density of the imbricated structure was dependent on the spray diameter during the ESD process, and the root-mean-square roughness of the film surface decreased with increasing applied voltage. These findings suggest that the ESD method will be effective for the preparation of fullerene-derivative thin films for the production of organic devices.
\end{abstract}

Keywords: Electrospray Deposition Method; Fullerene Derivative; Thin Film; Scanning Electron Microscope; Imbricated Structure; Atomic Force Microscope; Root-Mean-Square Roughness

\section{Introduction}

Organic devices are of interest owing to their special advantages over inorganic semiconductor devices, such as their light weight, flexibility, large device area, lowcost manufacture, and the applicability of solution-processing techniques. Solution processes have already been demonstrated with organic semiconductors for the fabriccation of light-emitting diodes [1], thin-film transistors [2], and photovoltaic cells [3]. On the contrary, a bilayer heterojunction photovoltaic system [4-6], in which donor and acceptor films are stacked sequentially on top of one another, is difficult to form practically with different organic compounds using normal solution processes such as spin-coating, dip-coating, screen printing, and inkjet printing. This is because these solution processes cause the dissolution of the underlying organic layer while coating the upper organic layer. In addition, the formation of a single layer of fullerene or a fullerene derivative through a solution process has innate drawbacks: the thickness and surface roughness cannot be controlled because of the poor film-forming ability due to strong

\footnotetext{
Corresponding author.
}

cohesive forces, and these species have low solubility in common organic solvents. Therefore, the considerable amount of research on the fabrication of fullerene films has dealt almost exclusively with as-deposited films, consisting of vacuum-sublimed $\mathrm{C}_{60}$ on top of a spincoated polymer film [4-6]. However, most fullerene derivatives cannot be deposited by vacuum processes, and there have been few reports on the fabrication of fullerene-derivative films by the spin-coating process [7]. For the fabrication of fullerene-derivative-based bilayer heterojunction photovoltaic devices, an appropriate solution process for fullerene-derivative thin-film deposition is desirable.

Recently, our research group has investigated a new electrospray deposition (ESD) method as a film-formation process for organic semiconductors, which can be adapted for the fabrication of organic photovoltaic (OPV) devices with regioregular poly(3-hexylthiophene-2,5-diyl) and fullerene derivatives [8]. In this ESD process, a high voltage of several kilovolts is applied to the organic solution, which is then divided into innumerable droplets with diameters of a few micrometers [9]. As a result, following solvent evaporation, organic semiconductor 
nanoparticles are formed by the agglomerating solute from the solution before it reaches the substrate. The ESD method is as good as the evaporation method for the formation of nanoparticulate films for successful multilayer fabrication without dissolution of the underlying organic layer. Furthermore, the method has the advantage that film formation is possible from very dilute organic semiconductor solutions (less than $100 \mu \mathrm{g} / \mathrm{mL}$ ).

To investigate how the homogeneous fullerene-derivative films are formed in this ESD process, we determined the controllable factors: the applied voltage and the spray diameter (signifying the spray angle of the electrospray). In this study, we show the electrospray deposition of films of several soluble fullerene derivatives that are well-known electron-acceptor materials for OPV devices, and discuss the relationship between the film-growth mechanisms of the fullerene derivatives and their molecular structures.

\section{Experimental}

\subsection{Materials}

$[6,6]-$ Phenyl- $\mathrm{C}_{61}$-butyric acid methyl ester (PC[60]BM) [10] and [6,6]-phenyl- $\mathrm{C}_{71}$-butyric acid methyl ester (PC[70]BM) [11] (purity, 99\%) were purchased from Frontier Carbon. Purified indene- $\mathrm{C}_{60}$-monoadduct (ICMA) [12] (99.9\%) was obtained from FLOX corp., and o-dichlorobenzene (o-DCB: Wako Pure Chemical Industries) and acetonitrile (Wako Pure Chemical Industries) were used as received.

\subsection{ESD Process}

The fullerene-derivative powder $(1.0 \mathrm{mg})$ was mixed with $o$-DCB $(1 \mathrm{~mL})$. Then, acetonitrile was added to the resulting solution as an additional solvent. The concentration of the additional solvent was 10 vol\%. Figure 1 shows the experimental setup for the ESD process. We used a glass capillary for the ESD process because of the controllability of its diameter, which leads to the formation of an organic thin film with a smooth surface [13]. The glass capillary (inner diameter $\approx 50 \mu \mathrm{m}$ ) was fabricated using a puller (Narishige PC-10) and a microforge (Narishige MF-900). A high-voltage source (Matsusada Precision HJPQ-30P1) was used to apply a positive high voltage to a copper wire in the fullerene-derivative solution in the glass capillary. The earthed line was connected to an indium tin oxide (ITO) layer on top of the glass substrate.

The ITO-coated glass substrate was cleaned in solvent (isopropyl alcohol, acetone, and ethanol) and deionized water with ultrasonic treatment. The spray diameter and supply rate of the ESD process were measured with a charge-coupled device (CCD) camera (Watec WAT-902B), as shown in Figure 1. The distance from the glass capil-

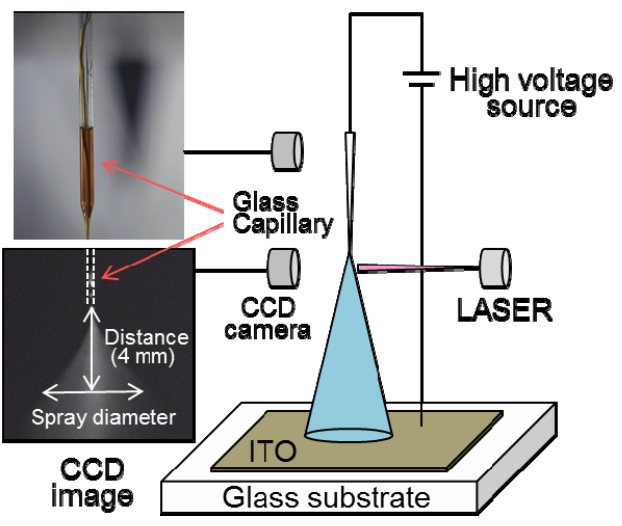

Figure 1. Experimental setup for ESD method.

lary to the substrate was $100 \mathrm{~mm}$, and the applied high voltage was varied from 3.5 to $8.0 \mathrm{kV}$. The surface roughness of the neat film was estimated by atomic force microscopy (AFM; Seiko SPA-300) and scanning electron microscopy (SEM; Hitachi Science Systems S-4100).

The dipole moments of the fullerene derivatives were determined by using the semiempirical PM3 Hamiltonian and the B3LYP/6-31G(d) method, as implemented in Gaussian 09.

\section{Results and Discussion}

\subsection{Film Formation by ESD Method}

In our previous study, we found that the dielectric constant of the solvent used in the ESD process influences the spray diameter significantly [14]. Solvents with low dielectric constants such as $o$-DCB showed relatively small spray diameters. However, the electrospray was spread significantly owing to the increasing Coulomb repulsion when another solvent with a high relative dielectric constant was added. As a result, the surface roughness of the organic thin film was also reduced drasticcally by the dense deposition of fine particles [15]. Therefore, we adopted the abovementioned mixed-solvent technique to achieve fullerene-derivative film deposition by the ESD method.

Solutions of three types of fullerene derivatives were prepared for investigation of the solute-structure dependence on the ESD conditions (Figure 2). The changes in supply rate of each solution to the glass capillary with the voltage applied during the spray deposition of the fullerene-derivative solutions are shown in Figure 3. The solution supply rate increased linearly with increasing voltage in all cases. The structures of the fullerene derivatives did not affect the slopes of the plots significantly.

Figure 4 shows the influence of the applied voltage on the spray diameter measured $4 \mathrm{~mm}$ below the tip of the glass capillary. In all cases, the spray diameter increased with increasing applied voltage, and reached a 


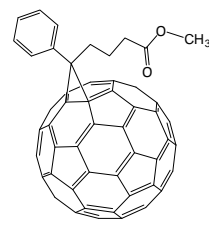

PC [60] BM

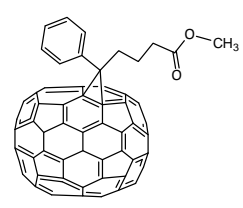

$\mathrm{PC}[70] \mathrm{BM}$

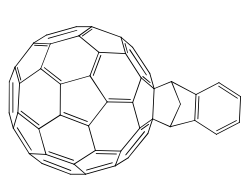

ICMA
Figure 2. Fullerene derivatives used in this study.

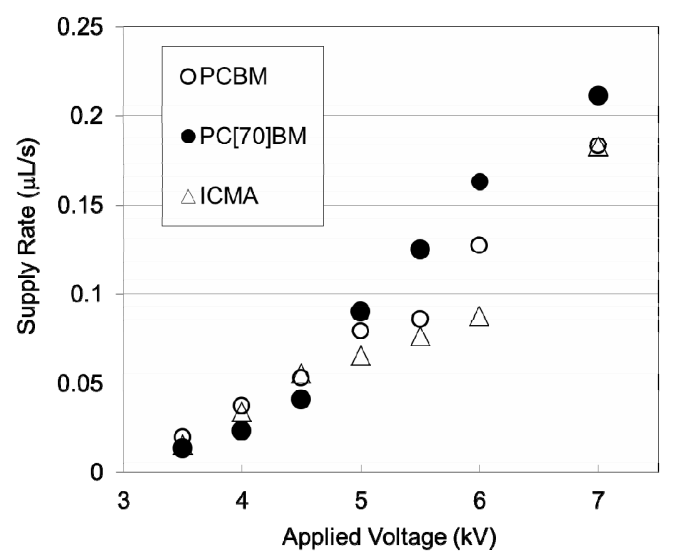

Figure 3. Influence of applied voltage on supply rate of fullerene solution.

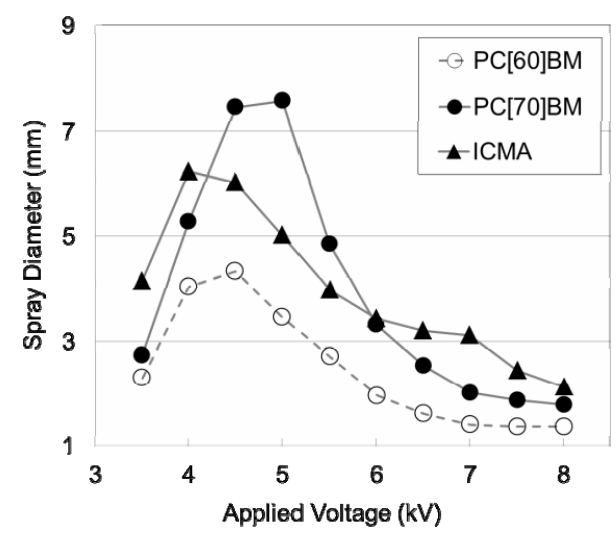

Figure 4. Spray diameter $\mathbf{4} \mathbf{~ m m}$ far from glass capillary as a function of applied high voltage.

maximum value at 4.0 (ICMA), 4.5 (PC[60]BM), or 5.0 $\mathrm{kV}(\mathrm{PC}[70] \mathrm{BM})$. These results imply that a stable Taylor cone was formed at the tip of the glass capillary at these voltages $\left(V_{\text {Dmax }}\right)$, causing the maximum spray diameter $\left(D_{\max }\right)$ to be achieved under these deposition conditions $[16,17]$. PC[60]BM, PC[70]BM, and ICMA had $D_{\max }$ values of $4.3,7.6$, and $6.2 \mathrm{~mm}$, respectively, at $V_{\text {Dmax }}$. It should be noted that $D_{\max }$ of PC[70]BM was almost double that of PC[60]BM. The $D_{\max }$ values of each fullerene derivative and their calculated dipole moments are summarized in Table 1. It is clear that the increase in $D_{\max }$ is due to the increase in the dipole moment. The dipole moments of the fullerene derivatives affect the charge densities of the droplets generated from the solution. Our previous study revealed that the spray diameter was directly proportional to the dielectric constant of the solvent. This is because with a high relative dielectric constant of the solvent, the polar surface of the droplets causes a spread in the spray diameter owing to the increased Coulomb repulsion [14]. These results indicate that the droplets containing fullerene derivatives showed their maximum spread at $V_{\text {Dmax }}$, and the Coulomb repulsion between droplets was at its maximum in the direction perpendicular to the movement of droplets toward the substrate.

\subsection{SEM and AFM Observations}

The fullerene-derivative films formed by the ESD process were observed by SEM. In all cases, an imbricated structure, formed by the stacking of fullerene-derivative pieces, was obtained in the vicinity of $V_{\text {Dmax }}$ (Figure 5). In the case of $\mathrm{PC}[60] \mathrm{BM}$, when the applied voltage was increased to $4.5 \mathrm{kV}$, the number of fullerene-derivative pieces in an area of $100 \times 100 \mu \mathrm{m}$ increased, reaching a maximum value of 120 , with an average piece size of about $10 \mu \mathrm{m}$. As the applied voltage was increased beyond $V_{\text {Dmax }}$, the number of pieces decreased to less than 100 in the same area. The average size of the PC[60]BM pieces was found to be greater than $10 \mu \mathrm{m}$ at $4.5 \mathrm{kV}$. The observations for PC[70]BM and ICMA were very similar to results in the case of PC[60]BM. Accordingly, it is supposed that the size of the imbricated pieces corresponds to the diameter of the droplets coming into contact with the substrate. At $6.0 \mathrm{kV}$, the imbricated structures were not observed, but the results showed aggregation defects of the fullerene derivatives PC[60]BM and PC[70]BM or no film area for ICMA. This was because the wet droplets came into contact with the substrate in high-voltage regions (high supply rate), resulting in agglutinated fullerene derivatives that were poorly adhered to the substrate.

For investigation of the surface nanostructures of the imbricated pieces, the fullerene-derivative films were observed by AFM in an area of $10 \times 10 \mu \mathrm{m}$ (Figure 6). The surface roughnesses of the deposited films were obtained from the AFM images; a planarizing surface was observed with increasing applied voltage for all samples. AFM observation of the ICMA and PC[70]BM films formed at $V_{\text {Dmax }}$ showed that their surfaces were comprised of 5 - $80 \mathrm{~nm}$ particles, but with PC[60]BM, no such nanoparticles were observed under any conditions (Figure 6). The nano surface structures were probably dependent on the self-organization behaviors of the fullerene derivatives. The relationship between the ESD applied voltage and the root-mean-square (RMS) roughnesses of the fullerene-derivative films is shown in Figure 7. It is noted that the RMS roughness of the surface was reduced dramatically around $V_{\text {Dmax }}$ to less 
Table 1. Summarized dipole moment, $D_{\max }$, and $V_{\text {Dmax }}$ values of fullerene derivatives.

\begin{tabular}{cccc}
\hline & dipole Moment $^{\mathrm{a}}($ debye $)$ & $D_{\max }(\mathrm{mm})$ & $V_{\text {Dmax }}(\mathrm{kV})$ \\
\hline PC[60]BM & 2.34 & 4.33 & 4.5 \\
ICMA & 3.15 & 6.27 & 4.0 \\
PC[70]BM & 3.32 & 7.97 & 5.0 \\
\hline
\end{tabular}

${ }^{a}$ Calculated the geometry and the electron density by DFT-method.; Gaussian 09 (RB3LYP/6-31G(d)).
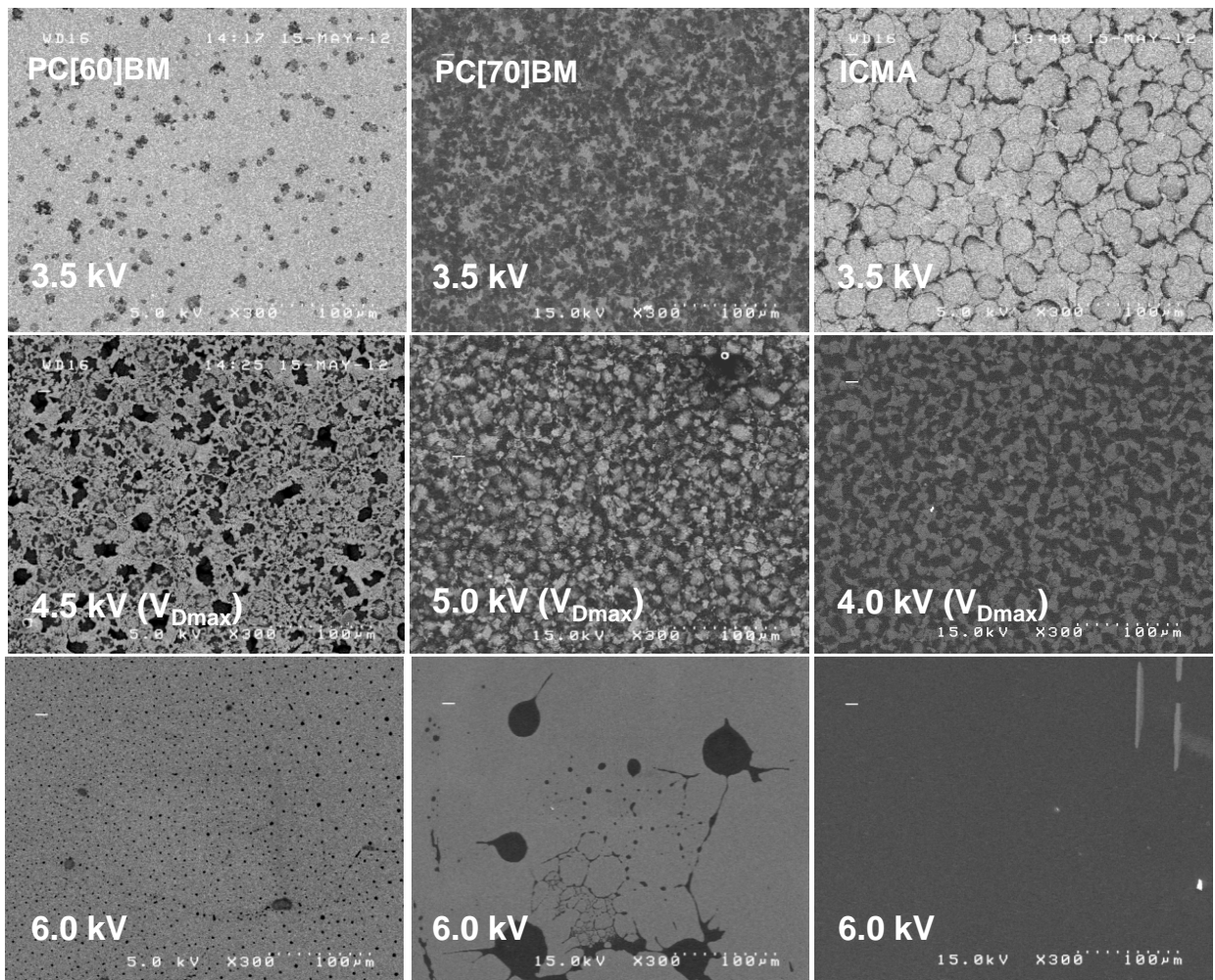

Figure 5. SEM images of fullerene derivatives thin films fabricated by ESD with several voltages; PC[60]BM (left), PC[70]BM (center) and ICMA (right).
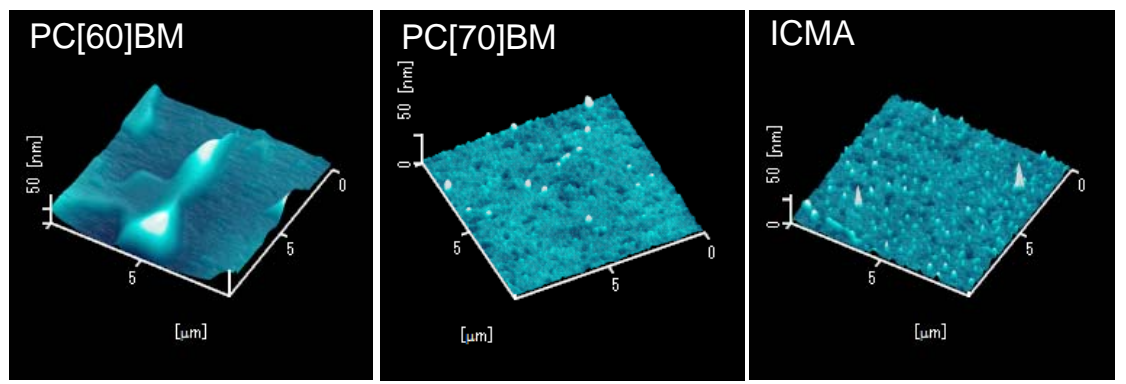

Figure 6. AFM images of fullerene derivatives thin films fabricated at $V_{\text {Dmax }}$ by ESD method.

than $10 \mathrm{~nm}$.

\section{Conclusions}

In conclusion, we investigated the film-forming properties of various fullerene derivatives in the ESD process, and obtained the following results:
1) The ESD process enables the fabrication of single films of various fullerene derivatives that are used as organic semiconductor materials.

2) The applied voltage giving the maximum spray diameter varies according to the fullerene derivative employed.

3) SEM studies showed that, at the maximum spray 


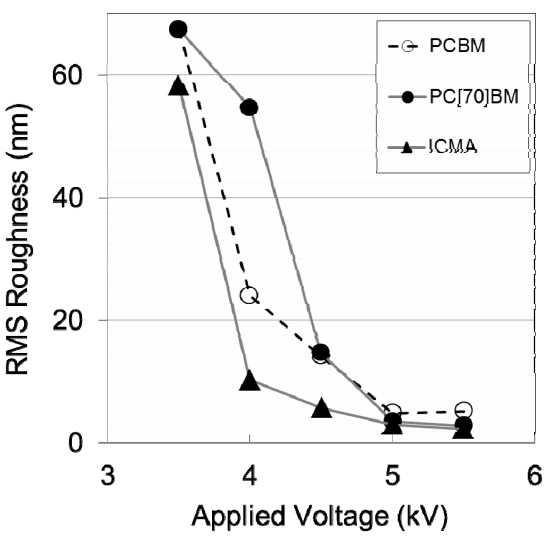

Figure 7. Relationship between applied voltage and RMS roughness estimated from AFM image.

diameter, the deposited films consist of the imbricated structure formed by the stacking of $10 \mu \mathrm{m}$ fullerene-derivative sheets.

4) The RMS roughness of the film surface drops sharply when the maximum spray diameter is reached.

\section{REFERENCES}

[1] J. H. Burroughes, D. D. C. Bradley, A. R. Brown, R. N. Marks, K. Mackay, R. H. Friend, P. L. Burns and A. B. Holmes, "Light-Emitting Diodes Based on Conjugated Polymers," Nature, Vol. 347, 1990, pp. 539-541. doi: $10.1038 / 347539 \mathrm{a} 0$

[2] H. Kawaguchi, S. Iba, Y. Kato, T. Sekitani, T. Someya and T. Sakurai, "A 3-D-Stack orgaNic Sheet-Type Scanner with Double-Wordline and Double-Bitline Structure," Sensors Journal, IEEE, Vol. 6, No. 5, 2006, pp. 12091217. doi:10.1109/JSEN.2006.881375

[3] M. Hiramoto, H. Fujiwara and M. Yokoyama, " $p-i-n$ Like Behavior in Three-Layered Organic Solar Cells Having a Codeposited Interlayer Of Pigments," Journal of Applied Physics, Vol. 72, No. 8, 1992, pp. 3781-3787. doi: $10.1063 / 1.352274$

[4] N. S. Sariciftci, D. Braun, C. Zhang, V. I. Srdanov, A. J. Heeger, G. Stucky and F. Wudl, "Semiconducting Polymer Buckminsterfullerene Heterojunctions: Diodes, Photodiodes, and Photovoltaic Cells," Applied Physics Letters, Vol. 62, No. 6, 1993, pp. 585-587. doi:10.1063/1.108863

[5] J. J. M. Halls, K. Pichler, R. H. Friend, S. C. Moratti and A. B. Holmes, "Exciton Diffusion and Dissociation in a Poly(p-phenylenevinylene) $/ \mathrm{C}_{60}$ Heterojunction Photovoltaic Cell," Applied Physics Letters, Vol. 68, No. 22, 1996, pp. 3120-3122. doi:10.1063/1.115797

[6] L. S. Roman, M. R. Andersson, T. Yohannes and O. Inganás, "High Quantum Efficiency Polythiophene $/ \mathrm{C}_{60}$ Photodiodes," Advanced Materials, Vol. 10, No. 10, 1998, pp. 774-777.

doi:10.1002/(SICI)1521-4095(199807)10:10<774::AIDADMA774>3.0.CO;2-J

[7] L. C. Chen, D. Godovsky, O. Inganás, J. C. Hummelen, R.
A. J. Janssens, M. Svensson and M. R. Andersson, "Polymer Photovoltaic Devices from Stratified Multilayers of Donor-Acceptor Blends," Advanced Materials, Vol. 12, No. 18, 2000, pp. 1367-1370. doi:10.1002/1521-4095(200009)

[8] T. Fukuda, K. Takagi, T. Asano, Z. Honda, N. Kamata, H. Shirai, J. Ju, Y. Yamagata and Y. Tajima, "Improved Power Conversion Efficiency of Organic Photovoltaic Cell Fabricated by Electrospray Deposition Method by Mixing Different Solvents," Japanese Journal of Applied Physics, Vol. 51, 2012, Article ID: 02BK12. doi:10.1143/JJAP.51.02BK12

[9] V. N. Morozov and T. Y. Morozova, "Electrospray Deposition as a Method to Fabricate Functionally Active Protein Films," Analytical Chemistry, Vol. 71, No. 7, 1999, pp. 1415-1420. doi:10.1021/ac9808775

[10] J. C. Hummelen, B. W. Knight, F. LePeq, F. Wudl, J. Yao and C. L. Wilkins, "Preparation and Characterization of Fulleroid and Methanofullerene Derivatives," The Journal of Organic Chemistry, Vol. 60, No. 3, 1995, pp. 532538. doi:10.1021/jo00108a012

[11] M. M. Wienk, J. M. Kroon, W. J. H. Verhees, J. Knol, J. C. Hummelen, P. A. Hal and R. A. J. Janssen, "Efficient Methano[70]fullerene/MDMO-PPV Bulk Heterojunction Photovoltaic Cells," Angewandte Chemie International Edition, Vol. 42, No. 29, 2003, pp. 3371-3375. doi:10.1002/anie.200351647

[12] A. Puplovskis, J. Kacens and O. Neilands, "New Route for [60]Fullerene Functionalisation in [4+2] Cycloaddition Reaction Using Indene," Tetrahedron Letters, Vol. 38 , No. 13 , 1997, pp. 285-288. doi:10.1016/S0040-4039(96)02293-9

[13] T. Fukuda, K. Takagi, T. Asano, Z. Honda, N. Kamata, K. Ueno, H. Shirai, J. Ju, Y. Yamagata and Y. Tajima, "Bulk Heterojunction Organic Photovoltaic Cell Fabricated by the Electrospray Deposition Method Using Mixed Organic Solvent," Physica Status Solidi (RRL)—Rapid Research Letters, Vol. 5, No. 7, 2011, pp. 229-231. doi:10.1002/pssr.201105232

[14] T. Fukuda, H. Asaki, T. Asano, K. Takagi, Z. Honda, N. Kamata, J. Ju and Y. Yamagata, "Surface Morphology of Fluorene Thin Film Fabricated by Electrospray Deposition Technique Using Two Organic Solvents: Application for Organic Light-Emitting Diodes," Thin Solid Films, Vol. 520, No. 1, 2011, pp. 600-605. doi:10.1016/j.tsf.2011.07.048

[15] J. Ju, Y. Yamagata and T. Higuchi, "Thin-Film Fabrication Method for Organic Light-Emitting Diodes Using Electrospray Deposition," Advanced Materials, Vol. 21, No. 43, 2009, pp. 4343-4347. doi:10.1002/adma.200900444

[16] H. Oh, K. Kim and S. Kim, "Characterization of Deposition Patterns Produced by Twin-Nozzle Electrospray," Journal of Aerosol Science, Vol. 39, No. 9, 2008, pp. 801-813. doi:10.1016/j.jaerosci.2008.05.003

[17] A. M. Gañán-Calvo, J. Dávila and A. Barrero, "Current and Droplet Size in the Electrospraying of Liquids. Scaling Laws," Journal of Aerosol Science, Vol. 28, No. 2, 1997, pp. 249-275. doi:10.1016/S0021-8502(96)00433-8 